OPEN ACCESS

Edited by:

Rosalia Crupi,

University of Messina, Italy

Reviewed by:

Silvia Vincenzetti,

University of Camerino, Italy

Si-Yang Huang,

Yangzhou University, China

*Correspondence:

Chun Lian Song

chunlian-song@outlook.com

Specialty section:

This article was submitted to

Veterinary Pharmacology and

Toxicology,

a section of the journal

Frontiers in Veterinary Science

Received: 14 May 2021 Accepted: 23 September 2021 Published: 12 November 2021

Citation:

Wang YL, Shu XH, Zhang X, Liu YB, Zhang YJ, LV T, Huang $X$ and Song $C L$ (2021) Effects of Two Polysaccharides

From Traditional Chinese Medicines

on Rat Immune Function.

Front. Vet. Sci. 8:703956

doi: 10.3389/fvets.2021.703956

\section{Effects of Two Polysaccharides From Traditional Chinese Medicines on Rat Immune Function}

\author{
Yu Lei Wang ${ }^{1}$, Xiang Hua Shu ${ }^{1}$, Xue Zhang ${ }^{1}$, Yong Bo Liu ${ }^{2}$, Ya Jing Zhang ${ }^{1}$, Tao Lv ${ }^{1}$, \\ Xin Huang ${ }^{1}$ and Chun Lian Song ${ }^{1 *}$ \\ ${ }^{1}$ College of Veterinary Medicine, Yunnan Agricultural University, Kunming, China, ${ }^{2}$ College of Veterinary Medicine, South \\ China Agricultural University, Guangzhou, China
}

\begin{abstract}
The aim is to study the immune function effect of two polysaccharides extracted from traditional Chinese herbs on rats. Ultrasonic-assisted extraction was used to extract the polysaccharide from traditional Chinese medicines. MTT assay was used to determine the effects of two polysaccharides on the conversion of pig peripheral $\mathrm{T}$ lymphocytes. For this, 24 Sprague-Dawley rats were selected for the clinical trial and divided into groups B (blank), CK (cyclophosphamide inhibitory control), AP (angelica polysaccharide), and RIP (radix isatidis polysaccharide). Except for group B, other groups can induce the immunodeficiency by using cyclophosphamide. Rats of the AP and RIP groups were given gavage of $1 \mathrm{~mL}$ of $\mathrm{AP}$ and RIP. The blood was sampled from the eyeball on days $0,7,14,21,28$, and 35 , respectively, to determine immune cells, IgG and IgM of immunoglobulin, body weight, and spleen index.
\end{abstract}

Results: The average content of AP and RIP was 51.27 and $14.8 \%$, and the extraction rate was 75.23 and $60.94 \%$. The maximum stimulation index was 1.407 when the concentration of AP was $8,000 \mu \mathrm{g} \mathrm{mL}^{-1}$ and 1.5 when the concentration of RIP was $125 \mu \mathrm{g} \mathrm{mL}^{-1}$. Both kinds of polysaccharides can alleviate the decline of white blood cells, lymphocytes, monocytes, neutrophils, and serum IgG and IgM caused by cyclophosphamide. The two polysaccharides can regulate the rapid recovery of weight in immunosuppressed rats and increase the spleen index of immunosuppressed SD rats. The polysaccharides from the two traditional Chinese medicines can alleviate the immunosuppression caused by cyclophosphamide and promote the immune function of the body, which can be used as raw material resources of new veterinary medicine.

Keywords: angelica, radix isatidis, polysaccharides, immune function, rats

\section{INTRODUCTION}

Angelica, first contained in Agriculture God's Canon of Materia Medica (Shennong Bencao Jing), is a common Chinese herbal medicine of the dried roots of Angelica (the umbelliferae plants) (1). Its main active components include ferulic acid, ligustilide and their isomers, and polysaccharides (2-4). Modern pharmacological studies show that AP can improve immunity and show antitumor, antiradiation, antioxidation, and antiaging effects (5-7). Radix isatidis, the dried root of the isatis tinctoria (Cruciferous plant), recorded in Agriculture God's Canon of Materia Medica, has the effect of clearing heat and detoxifying, cooling blood, and tonifying the pharynx (8). Modern 
pharmacology indicates that radix isatidis has antiviral, anti-inflammatory, and antipyretic effects (9-11), and radix isatidis polysaccharide (RIP) is its major component (12). Polysaccharide from traditional Chinese medicine can improve the immunity of animals (13), and it is also reported to enhance the phagocytosis of the mono-macrophage system, activate the reticuloendothelium system, induce the proliferation of lymphocytes, recognize antigens, and improve the lethality of NK cells (14-16). Antitumor and chemical protective effects of ginseng polysaccharide have received a lot of attention. In a tumor-bearing mouse model, a sublethal dose of cyclophosphamide after treatment with $100 \mathrm{mg} / \mathrm{kg}$ Ganshan injection significantly reduced mortality and promoted recovery (17).

However, with the vigorous development and continuous innovation of animal husbandry, the extensive use of antibiotics in animal husbandry production has brought a huge threat to food and environmental safety. The issue of drug-resistant bacteria has been raised many times by the World Health Organization. In the future, antibiotics as animal feed additives will be controlled more strictly, which will become the biggest obstacle to the future development of animal husbandry (18). Therefore, two kinds of polysaccharides were extracted from traditional Chinese medicine in this experiment and used to explore their effect on the immune function of cyclophosphamide immunosuppressed rats so as to provide relevant experimental data for the development of veterinary drugs.

\section{MATERIALS AND METHODS}

\section{Ethics Statement}

All animal protocols were approved and supervised by the Experimental Animal Ethics Committee of YunNan Agricultural University NO. YNAU2019llwyh012.

\section{Experimental Animals and Medicinal Materials}

A total of 24 Sprague-Dawley (SD) rats, weighting $150 \pm 10 \mathrm{~g}$, equal in gender, were purchased from the SPF Experimental Animal Center of Kunming Medical University. Landrace, weighing 20-30 kg, were purchased from the Experimental Pig Farm of Yunnan Agricultural University.

Angelica and radix isatidis were purchased from the traditional Chinese medicinal material market in Yunnan Province and were identified and qualified by the associate professor Bai Weibing of Yunnan Agricultural University. Angelica originated from Qujing, Yunnan, and radix isatidis originated from Luxi County, Honghe Prefecture, Yunnan.

This experiment has been approved by the Animal Ethics Committee of Yunnan Agricultural University.

\section{Test Reagents and Instruments}

Test reagents included IL-2 kit (Lot No. 9670032121, ABclonal), IgG kit (Lot No. 9670012721, ABclonal), MTT (Lot No. 3580MG250, Biofrox), Erythrocyte lysate (Lot No. 67106955, Biosharp), fetal bovine serum (Lot No. 1828728, Gibco), and pig peripheral lymphocyte separation medium KIT (Lot No.
LTS1110, Tianjin Hao Yang Biological Products Technology Co., LTD.)

Instruments included ultrasonic cleaning agent (JP-010D), Skymen cleaning equipment (Shenzhen Co., Ltd.), ultraviolet spectrophotometer (MODEL: EU-2600R, Shanghai Onlab Instrument Co., Ltd.), veterinary blood cell analyzer (MODEL BC-2800VET), carbon dioxide incubator (MODEL MCO-15AC, Sanyo Electric Co., Ltd).

\section{Experimental Methods}

\section{Extraction and Content Determination of} Polysaccharide From Angelica and Radix Isatidis

The dried radix angelica medicinal material was crushed by a pulverizer and then passed through a 40 -mesh screen to obtain powdered medicine. A total of $10 \mathrm{~g}$ powder was taken and added to $400 \mathrm{~mL}$ deionized water. Then, the mix was given ultrasonic extraction at $60^{\circ} \mathrm{C}$ for $15 \mathrm{~min}$ and then placed in a water bath at $80^{\circ} \mathrm{C}$ for $1 \mathrm{~h}$. The mix was concentrated to $50 \mathrm{~mL}$ by rotary evaporation and added to $267 \mathrm{ml}$ of $95 \%$ ethanol to make the alcohol content $80 \%$ and remained in a refrigerator overnight at $4^{\circ} \mathrm{C}$. The solution was centrifuged at 2,000 $\mathrm{min}^{-1}$. The supernatant was taken, dried at $45^{\circ} \mathrm{C}$, and stored at $4^{\circ} \mathrm{C}$. The extraction method of RIP was the same as above. The content of polysaccharides was determined by phenol sulfuric acid assay. Briefly, accurately weigh $10 \mathrm{mg}$ of glucose standard into a $250-\mathrm{mL}$ volumetric flask, add double distilled water to the mark, and draw $0.2,0.3,0.4,0.5,0.6,0.7,0.8$, and $0.9 \mathrm{~mL}$, respectively, with distilled water to make up to $1.0 \mathrm{~mL}$, add $1 \mathrm{~mL}$ of $5 \%$ phenol and $5.0 \mathrm{~mL}$ of concentrated sulfuric acid, shake well and cool down, and then measure the optical density at $490 \mathrm{~nm}$ by ultraviolet spectrophotometer after standing at room temperature for $20 \mathrm{~min}$. The $1.0 \mathrm{ml}$ of water performed as the blank control group. The standard curve was made with polysaccharide weight as abscissa and optical density value as ordinate. The regression equation was $\mathrm{Y}=14.031 \mathrm{X}-0.002\left(R^{2}\right.$ $=99.75 \%$ ), indicating a good linear relationship and accessible standard curve.

\section{Transformation Experiment of T Lymphocyte}

Landrace were adapted to the environment for $3 \mathrm{~d}$ and were taken for blood samples via the anterior vena cava. The peripheral blood lymphocytes of pigs were separated according to the instructions of the Tianjin Haoyang Lymphocyte Separation Kit (http://www.tbdscience.com/ProductsDetail.aspx?para=6075A317BF-BEB1-4943-A8FF-4FF07F0B7D0D). Trypan blue staining was used for cell count (observation under microscopy of 40X). Then, lymphocytes proliferation experiment was performed. In detail, lymphocytes were inoculated evenly into a 96-well cell plate; each well contains about $10^{5}-10^{6}$ cells. A total of four experimental groups were set up (I, II, III, IV), and each experimental group was repeated four times.

Cell suspension and different concentrations of TCM polysaccharide $(31.25-8,000 \mu \mathrm{g} / \mathrm{mL})$ were added to group I. In group II, only cell suspension was added. RPMI1640 complete culture medium and different concentrations of TCM polysaccharide were added to group III. In group IV, only RPMI1640 complete medium was added. Continue to incubate 
TABLE 1 | Experimental grouping

\begin{tabular}{ll}
\hline Groups & Treatments \\
\hline Group I & Cell suspension, different concentrations of TCM polysaccharide \\
Group II & Cell suspension \\
Group III & RPMl1640 complete culture medium, different concentrations of \\
& TCM polysaccharide \\
Group IV & RPMl1640 complete culture medium
\end{tabular}

TABLE 2 | Experimental animal grouping.

\begin{tabular}{ll}
\hline Groups & Treatments \\
\hline Group B & $\begin{array}{l}\text { The rats were given intraperitoneal injection of normal saline on } \\
\text { days 4, 5, and } 6 \text { and gavage of drinking water on day } 4 .\end{array}$ \\
Group CK & $\begin{array}{l}\text { The rats were given intraperitoneal injection of CTX on days 4, 5, } \\
\text { and } 6 \text { and gavage of drinking water on day } 4 .\end{array}$ \\
Group AP & $\begin{array}{l}\text { The rats were given intraperitoneal injection of CTX on days 4, 5, } \\
\text { and } 6 \text { and gavage of angelica polysaccharide on day } 4 .\end{array}$ \\
Group RIP & $\begin{array}{l}\text { The rats were given intraperitoneal injection of CTX on days 4, 5, } \\
\text { and } 6 \text { and gavage of RIP on day } 4\end{array}$
\end{tabular}

The concentration of cyclophosphamide (CTX) was $70 \mathrm{mg} \cdot \mathrm{kg}^{-1} \cdot \mathrm{BW}$, dose of $1 \mathrm{~mL}$, once per day. The dose concentrations of RIP and AP were $200 \mathrm{mg} \cdot \mathrm{kg}^{-1} \cdot \mathrm{BW}$, dose of $1 \mathrm{~mL}$, once per day.

for $44 \mathrm{~h}$ under $5 \% \mathrm{CO}_{2}$, and then, add $5 \mu \mathrm{L}$ of MTT to each well under dark conditions. After continuing to cultivate for $4 \mathrm{~h}$, then measure the absorbance value at $570 \mathrm{~nm}$ by UV spectrophotometer. The experiment was repeated three times. Transformation experiment of T lymphocyte.

Stimulus Index (SI) were calculated as follows:

$$
S I=\left\{\left(O D_{\mathrm{I}}-O D_{\mathrm{III}}\right) /\left(O D_{\mathrm{II}}-O D_{\mathrm{IV}}\right)\right\} \times 100 \%
$$

Experimental groups are shown in Table 1.

\section{Rat Clinical Trails}

Experimental groupings and treatment are shown in Table 2. On days $0,7,14,21,28$, and $35,2 \mathrm{~mL}$ of blood was collected from the eyeball vein of SD rats in each group to detect IgG and IgM. Anticoagulant tubes of $0.5 \mathrm{~mL}$ were used to collect $100 \mu \mathrm{L}$ of blood from the eyeball vein to detect routine blood indicators. The SD rats were weighed before each blood collection and put to death after the last blood sampling, and their spleens were weighed and spleen index was calculated. Spleen index $=($ spleen weight/ rat weight) $\times 100 \%$.

\section{Statistical Analysis of Data}

All data were recorded and preliminarily processed with an Excel database and then analyzed by SPSS19.0 analysis software. The data results are expressed as mean \pm standard deviation. Analyze the difference of the parameters through the method of analysis of variance. The significant level of difference is $P>0.05$ or $P<0.01$.
TABLE 3 | Extraction rate and content of traditional Chinese medicine polysaccharide.

\begin{tabular}{lcccc}
\hline TCM & No. & Content (\%) & Average content (\%) & Extraction rate (\%) \\
\hline Angelica & 1 & 50.92 & $51.27 \pm 0.21$ & 75.23 \\
& 2 & 51.65 & & \\
& 3 & 51.24 & & 60.94 \\
Radix Isatidis & 1 & 15.09 & $14.80 \pm 0.23$ & \\
& 2 & 14.97 & & \\
& 3 & 14.34 & &
\end{tabular}

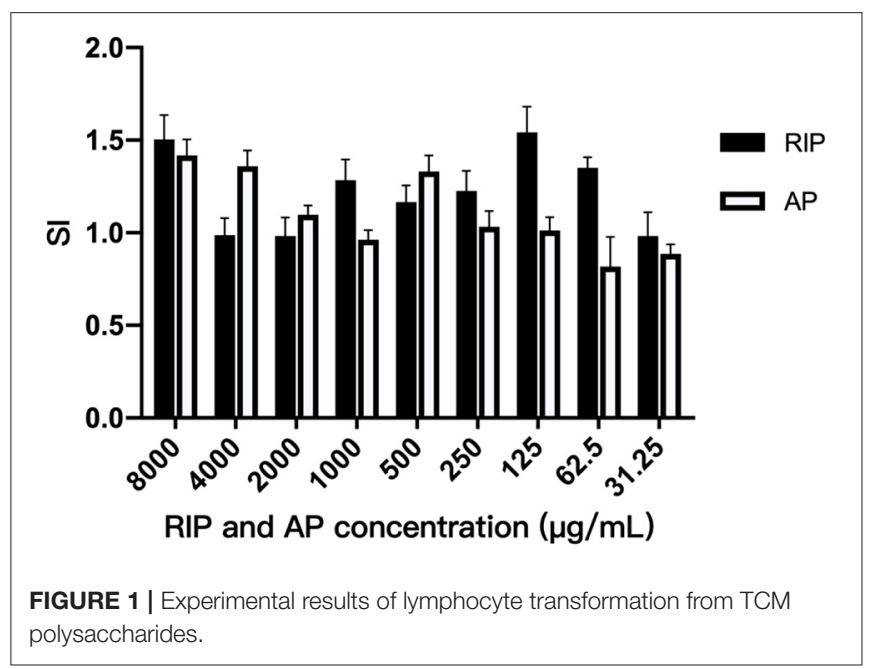

\section{RESULTS AND ANALYSIS}

\section{Extraction Rate and Content Results of Polysaccharide From Traditional Chinese Medicine}

Table 3 shows that AP content was $51.27 \pm 0.21 \%$ with the extraction rate of $75.23 \%$, and RIP was $14.80 \pm 0.23 \%$ with the extraction rate of $60.94 \%$.

\section{Experiment Results of T Lymphocyte Transformation}

It can be seen from Figure 1 that the SI were all greater than 1 when the concentration of AP was between $8,000 \mu \mathrm{g} / \mathrm{mL}$ and $250 \mu \mathrm{g} / \mathrm{mL}$, indicating that the AP at the above concentration could improve the transformation rate of $\mathrm{T}$ lymphocyte. The highest SI was 1.42 at $8,000 \mu \mathrm{g} / \mathrm{mL}$. SI was all greater than 1 when the concentration of RIP was between $8,000 \mu \mathrm{g} / \mathrm{mL}$ and $62.50 \mu \mathrm{g} / \mathrm{mL}$, indicating that the T lymphocyte conversion could be improved by RIP at the above concentration. The SI was up to 1.5 at $125 \mu \mathrm{g} / \mathrm{mL}$.

\section{Results of the Rat Clinical Trial}

\section{Effects of TCM Polysaccharides on Immune Cells}

It can be seen in Figure 2, that white blood cells (WBCs), Lym (lymphocyte), Mon (monocyte), and Gran (granulocyte) of the group CK, and the TCM polysaccharide groups were all declining after cyclophosphamide injection, reaching the lowest value on 

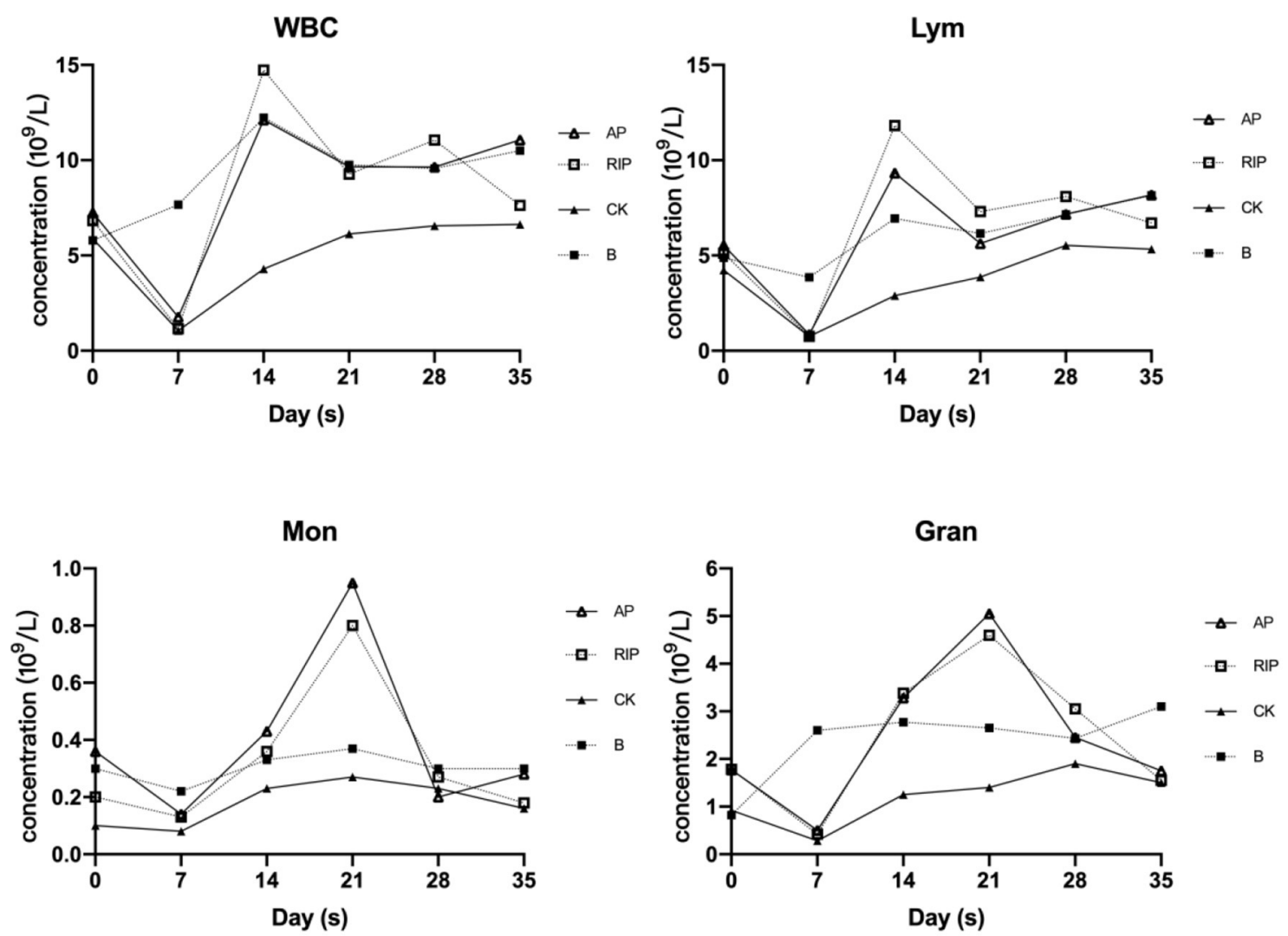

FIGURE 2 | Changes of WBC, Lym, Mon, and Gran.
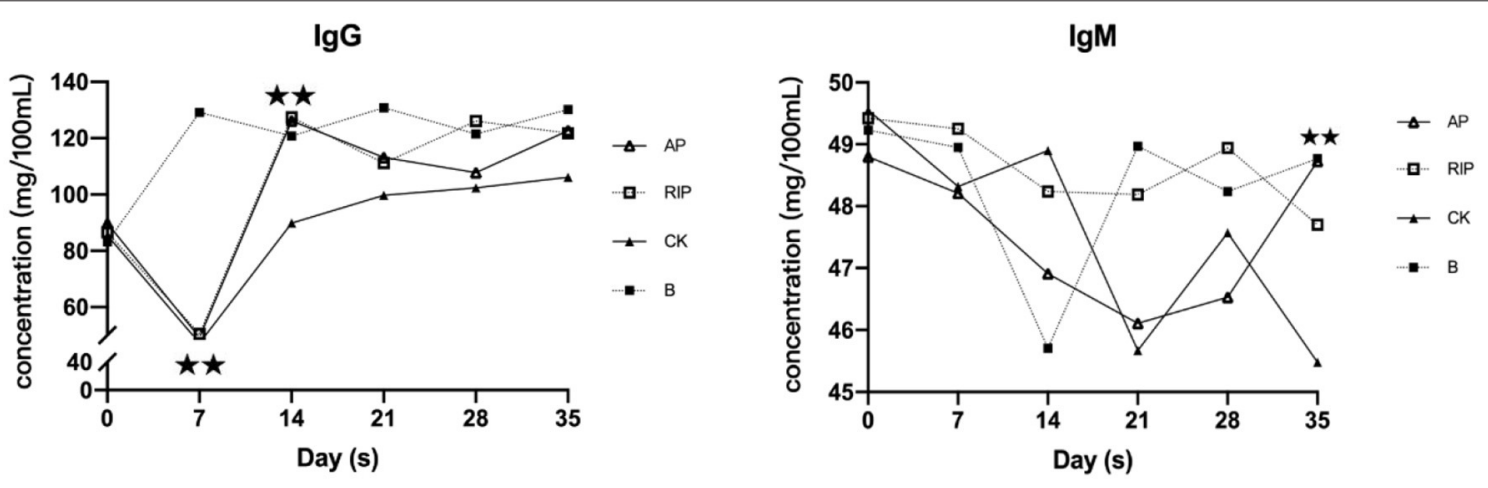

FIGURE 3 | Changes of immunoglobulin $\mathrm{G}(\mathrm{IgG})$ and IgM. $\star$ showed $P<0.05$, meaning there is significance; $\star \star$ showed $P<0.01$, meaning there is extreme significance.

day 7 , and then began to rise. The group CK was lower than group $\mathrm{B}$ and TCM polysaccharide groups at each time point. The TCM polysaccharide groups played an obvious promoting role on the amount of WBC, Lym, Mon, and Gran.

\section{Effects of TCM Polysaccharides on Immunoglobulin}

As shown in Figure 3, IgG in group CK was decreased first and then increased. IgG of TCM groups and group CK were all significantly lower than that of group $\mathrm{B}(P<0.01)$ on day 7. On the 14 th day, IgG of the TCM groups and group B were significantly higher than that of group CK $(P<0.01)$, and IgG of the two TCM groups returned to the normal level after 28 days. On day 28, the IgM indexes of groups AP and RIP were significantly higher than that of group CK $(P<0.01)$.

\section{Effects of TCM Polysaccharides on the Body Weight of SD Rats}

The body weight of SD rats in each experimental group showed an overall trend of increase as shown in Figure 4. There were significant differences between the TCM groups and group CK 


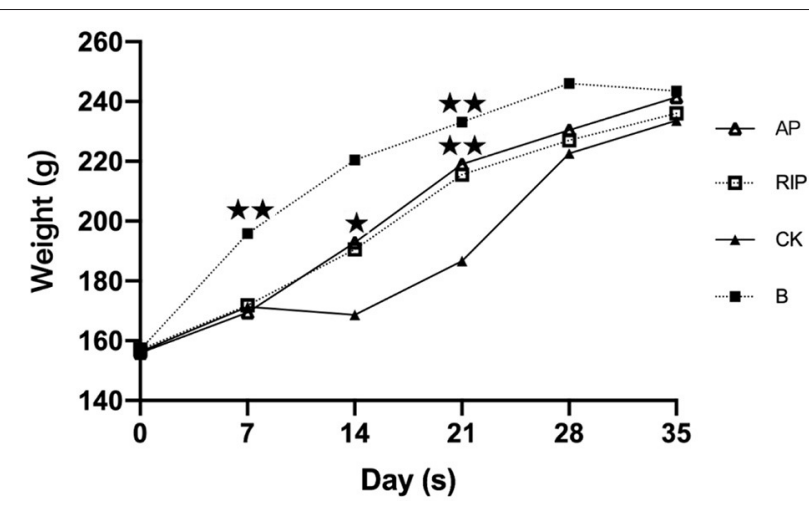

FIGURE 4 | Comparison of the weight. $\star$ showed $P<0.05$, meaning there is significance; $\star \star$ showed $P<0.01$, meaning there is extreme significance.

with group $\mathrm{B}$ in body weights on day $7(P<0.01)$. On day 14 , there were significant differences between the groups AP, RIP, and CK $(P<0.05)$. On day 21 , the body weight of group CK was significantly different from that of the other groups $(P<0.01)$. On day 35 , there was no significant difference among groups in the weight of SD rats $(P>0.05)$, indicating that the weight of immunosuppressed rats had returned to the normal level.

\section{Influence of TCM Polysaccharides on Spleen Index}

As shown in Figure 5, the spleen index of groups B, AP, and RIP were all higher than that of group CK. Compared with group $\mathrm{CK}$, there were significant differences in group RIP $(P<0.05)$ and extremely significant differences in group $\mathrm{B}(P<0.01)$. The results show that two kinds of TCM polysaccharides could increase the spleen index of immunosuppressed SD rats.

\section{DISCUSSION}

According to the report of Hou et al., AP, as the effective component of angelica, could significantly improve the red blood cell count and hemoglobin content of blood-deficient chickens. It could directly enrich the blood to increase red blood cells and hemoglobin, and it also regulates the hematopoietic factor, indirectly enriching the blood (19). According to Wang, the concentration of AP at $5 \sim 125 \mu \mathrm{g} / \mathrm{mL}$ can inhibit lymphatic proliferation induced by Concanavalin A. Serum transaminase (ALT and AST) levels were significantly reduced, and liver $\mathrm{H} \& \mathrm{E}$ staining showed that liver inflammation was reduced, indicating that ASP had potential liver-protection effects. ASP pretreatment could reduce liver injury caused by Concanavalin A in mice through anti-inflammatory and antioxidant effects (20). Du report the cell protection, antioxidant, and antiinflammatory effects of RIP on mouse alveolar macrophages stimulated by lipopolysaccharide (LPS). Pretreatment with RIP could significantly prolong the survival time of mouse alveolar macrophages and inhibit the generation of reactive oxygen species (ROS) and lipid peroxidation of alveolar macrophages after LPS stimulation (11). Zhao finds that RIP can enhance non-specific immune function, humoral immunity, and cellular

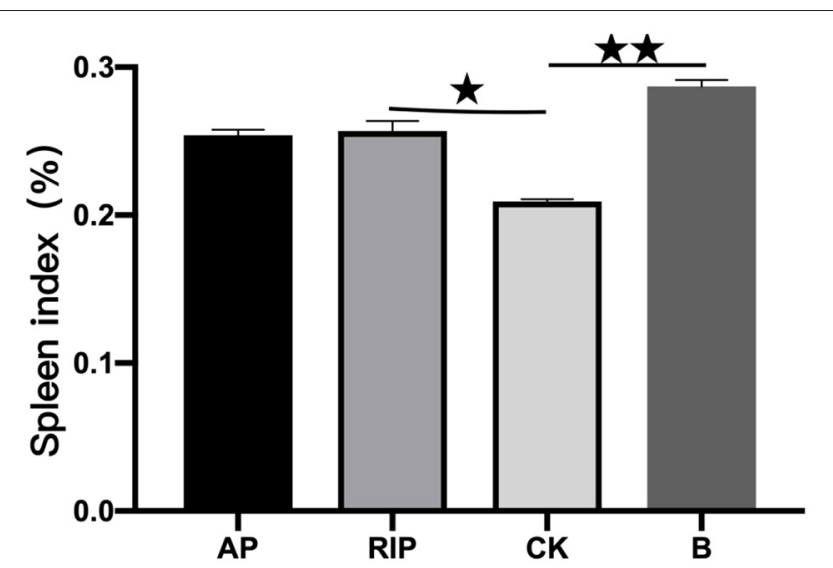

FIGURE 5 | Comparison of the spleen index. $\star$ showed $P<0.05$, meaning there is a significant difference compared with the RIP and CK groups; $\star \star$ showed $P<0.01$, meaning there is an extremely significant difference compared with the RIP and CK groups.

immunity in mice (21). These reports indicate that TCM polysaccharides have an enhanced effect on immunity that was similar to the results of this experiment.

Cyclophosphamide can degrade the activity of blood cell precursor in the bone marrow and reduce the number of red and white blood cells, thus leading to anemia, which is one of the main causes of low immunity (22). In this experiment, the number of red blood cells and hemoglobin in the two TCM groups were both increased to different degrees to enhance the immunity of immunosuppressed SD rats.

The body weight of the rats in the experimental group was on the rise, and the weight of group B was higher than those of the TCM groups and group CK. This indicates that cyclophosphamide affected the weight of SD rats, maybe due to the immunosuppression that affects appetite. Between days 14 and 21, the body weight of each drug group was higher than that of group $\mathrm{CK}$, indicating that these two polysaccharides can eliminate the inhibiting effect of cyclophosphamide on the body weight of SD rats to a certain extent.

The results of the experiment show that RIP can increase the spleen index of immunosuppressed SD rats and improve their immunity. Spleen weight is closely related to the body's immunity (23), and spleen index refers to the ratio of spleen weight to body weight. Li et al. report that dendrobium polysaccharides can enhance the immune function of spleen immunity by increasing spleen index and phagocytosis of macrophages and stimulating lymphocyte proliferation in mice (24).

The maximum lymphocyte SI was 1.407 when the concentration of AP was $8,000 \mu \mathrm{g} \mathrm{mL}^{-1}$. SI was greater than 1.5 when the concentration of RIP was $8,000 \mu \mathrm{g} \mathrm{mL}^{-1}$ and $125 \mu \mathrm{g} \mathrm{mL}^{-1}$. The results show that the two polysaccharides increased the $\mathrm{T}$ lymphocyte conversion rate at the above conditions. At the same time, clinical experimental results also show that the two polysaccharides could significantly alleviate the decline of RBC, WBC, lymphocytes, neutrophils, and serum IgG caused by cyclophosphamide. The results show that the two 
polysaccharides could alleviate the immunosuppression caused by cyclophosphamide and promote the immune function of the body.

\section{CONCLUSIONS}

Both AP and RIP can regulate the immune function of rats and have a value in new drug development and research.

\section{DATA AVAILABILITY STATEMENT}

The original contributions presented in the study are included in the article/supplementary material, further inquiries can be directed to the corresponding author/s.

\section{ETHICS STATEMENT}

The animal study was reviewed and approved by Animal Ethics Committee of Yunnan Agricultural University.

\section{AUTHOR CONTRIBUTIONS}

YW, XS, and CS conceived and designed the experiments. $\mathrm{YW}, \mathrm{XZ}$, and YL performed the experiments. YW,

\section{REFERENCES}

1. Zhao KJ, Dong TT, Tu PF, Song ZH, Lo CK, Tsim KW, et al. Molecular genetic and chemical assessment of radix Angelica (Danggui) in China. J Agric Food Chem. (2003) 51:2576-83. doi: 10.1021/jf026178h

2. Ma J, Huang J, Hua S, Zhang Y, Zhang Y, Li T, et al. The ethnopharmacology, phytochemistry and pharmacology of Angelica biserrata - A review. J Ethnopharmacol. (2019) 231:152-69. doi: 10.1016/j.jep.2018.10.040

3. Wei WL, Zeng R, Gu CM, Qu Y, Huang LF. Angelica sinensis in China-A review of botanical profile, ethnopharmacology, phytochemistry and chemical analysis. J Ethnopharmacol. (2016) 190:116-41. doi: 10.1016/j.jep.2016.05.023

4. Chen XP, Li W, Xiao XF, Zhang LL, Liu CX. Phytochemical and pharmacological studies on Radix Angelica sinensis. Chin J Nat Med. (2013) 11:577-87. doi: 10.1016/S1875-5364(13)60067-9

5. Wilasrusmee C, Kittur S, Siddiqui J, Bruch D, Wilasrusmee S, Kittur DS. In vitro immunomodulatory effects of ten commonly used herbs on murine lymphocytes. I Alternat Complement Med. (2002) 8:46775. doi: 10.1089/107555302760253667

6. Okuyama T, Takata M, Takayasu J, Hasegawa T, Tokuda H, Nishino A, et al. Anti-tumor-promotion by principles obtained from Angelica keiskei. Planta Med. (1991) 57:242-6. doi: 10.1055/s-2006-960082

7. Enoki T, Ohnogi H, Nagamine K, Kudo Y, Sugiyama K, Tanabe M, et al. Antidiabetic activities of chalcones isolated from a Japanese Herb, Angelica keiskei. J Agric Food Chem. (2007) 55:6013-7. doi: 10.1021/jf070720q

8. National Pharmacopoeia Committee. Chinese Pharmacopoeia (2010).

9. Liu Y, Chen M, Guo Q, Li Y, Jiang J, Shi J. Aromatic compounds from an aqueous extract of "Ban Lan Gen" and their antiviral activities. Acta Pharmaceut Sin B. (2017) 7:179-84. doi: 10.1016/j.apsb.2016.09.004

10. Ni LJ, Zhang LG, Hou J, Shi WZ, Guo ML. A strategy for evaluating antipyretic efficacy of Chinese herbal medicines based on UV spectra fingerprints. $J$ Ethnopharmacol. (2009) 124:79-86. doi: 10.1016/j.jep.2009.04.006

11. Du Z, Liu H, Zhang Z, Li P. Antioxidant and anti-inflammatory activities of Radix Isatidis polysaccharide in murine alveolar macrophages. Int J Biol Macromol. (2013) 58:329-35. doi: 10.1016/j.ijbiomac.2013.04.037
$\mathrm{YZ}, \mathrm{TL}$, and $\mathrm{XH}$ analyzed the experiments. YL and YW contributed to the reagents and materials and wrote the paper. All authors read and approved the manuscript.

\section{FUNDING}

This work was supported by Science and Technology Innovation Center Demonstration Plan (Kunming Science and Technology Innovation Center for Animal Epidemic Prevention and Control, KKJZ No. 2019-1-N-25318000003525). A major science and technology project in Yunnan Province Construction and Application of Technical System for Prevention and Control of Important Pig Diseases in Yunnan Province (202102AE090007).

\section{ACKNOWLEDGMENTS}

I would like to express my gratitude to all those who have helped me during the writing of this thesis. I gratefully acknowledge the help of my supervisor CS. I do appreciate her patience, encouragement, and professional instructions during my thesis writing.

12. Zhou W, Zhang XY. Research progress of Chinese herbal medicine radix isatidis (Banlangen). Am J Chin Med. (2013). 41:743-64. doi: 10.1142/S0192415X1350050X

13. Yu Y, Shen M, Song Q, Xie J. Biological activities and pharmaceutical applications of polysaccharide from natural resources: a review. Carbohydrate Polymers. (2018) 183:91-101. doi: 10.1016/j.carbpol.2017.12.009

14. Liu CP, Zhang X, Tan QL, Xu WX, Zhou CY, Luo M, et al. NF-кB pathways are involved in M1 polarization of RAW 264.7 macrophage by polyporus polysaccharide in the tumor microenvironment. PLoS ONE. (2017) 12:e188317. doi: 10.1371/journal.pone.0188317

15. Gong AG, Zhang LM, Lam CT, Xu ML, Wang HY, Lin HQ, et al. Polysaccharide of Danggui Buxue Tang, an ancient Chinese herbal decoction, induces expression of pro-inflammatory cytokines possibly via activation of NFKB signaling in cultured RAW 264.7 cells. Phytotherapy Res. (2017) 31:274-83. doi: $10.1002 /$ ptr.5745

16. Li ZP, Liu HB, Zhang QW, Li LF, Bao WR, Ma DL, et al. Interference of quercetin on astragalus polysaccharide-induced macrophage activation. Molecules. (2018) 23:1563. doi: 10.3390/molecules 23071563

17. T Tang HL, Chen C, Wang SK, Sun GJ. Biochemical analysis and hypoglycemic activity of a polysaccharide isolated from the fruit of Lycium barbarum L. Int J Biol Macromol. (2015) 77:235-42. doi: 10.1016/j.ijbiomac.2015.03.026

18. Machowska A, Lundborg C. Drivers of irrational use of antibiotics in Europe. Int J Environ Res Public Health. (2018) 16:27. doi: 10.3390/ijerph16010027

19. Hou H, Bao Y, Li Q, Shi W. Preparation of blood-deficient model and research of angelica polysaccharide on enriching blood in chickens. Evid Based Complement Alternat Med. (2012) 2012:965-47. doi: 10.1155/2012/965947

20. Wang K, Song Z, Wang H, Li Q, Cui Z, Zhang Y. Angelica sinensis polysaccharide attenuates concanavalin A-induced liver injury in mice. Int Immunopharmacol. (2016) 31:140-8. doi: 10.1016/j.intimp.2015.12.021

21. Zhao YL, Wang JB, Shan LM, Jin C, Ma L, Xiao XH. Effect of Radix isatidis polysaccharides on immunological function and expression of immune related cytokines in mice. Chin J Integr Med. (2008) 14:20711. doi: $10.1007 /$ s11655-008-0207-2 
22. Turk JL, Parker D. Effect of cyclophosphamide on immunological control mechanisms. Immunol Rev. (1982) 65:99-113. doi: 10.1111/j.1600-065X.1982.tb00429.x

23. Tian S, Hao C, Xu G, Yang J, Sun R. Optimization conditions for extracting polysaccharide from Angelica sinensis and its antioxidant activities. J Food Drug Anal. (2017) 25:766-75. doi: 10.1016/j.jfda.2016.08.012

24. Han J, Jiang X, Zhang L. Optimisation of extraction conditions for polysaccharides from the roots of Isatis tinctoria $\mathrm{L}$. by response surface methodology and their in vitro free radicals scavenging activities and effects on IL-4 and IFN- $\gamma$ mRNA expression in chicken lymphocytes. Carbohydrate Polymers. (2011) 86:1320-6. doi: 10.1016/j.carbpol.2011.06.036

Conflict of Interest: The authors declare that the research was conducted in the absence of any commercial or financial relationships that could be construed as a potential conflict of interest.
Publisher's Note: All claims expressed in this article are solely those of the authors and do not necessarily represent those of their affiliated organizations, or those of the publisher, the editors and the reviewers. Any product that may be evaluated in this article, or claim that may be made by its manufacturer, is not guaranteed or endorsed by the publisher.

Copyright $\odot 2021$ Wang, Shu, Zhang, Liu, Zhang, Lv, Huang and Song. This is an open-access article distributed under the terms of the Creative Commons Attribution License (CC BY). The use, distribution or reproduction in other forums is permitted, provided the original author(s) and the copyright owner(s) are credited and that the original publication in this journal is cited, in accordance with accepted academic practice. No use, distribution or reproduction is permitted which does not comply with these terms. 